\title{
Do We Need a Global Brain?
}

\author{
Jan Sliwa
}

\author{
Bern University of Applied Sciences, Biel, Switzerland, jan.sliwa@bfh.ch
}

\begin{abstract}
The new trend of Pervasive Computing, based on massively deployed wireless sensor and actuator networks, will enable gathering data about the world with an unprecedented accuracy, and using them to influence this world - both human beings and their environment. Among many application fields, the health support system will permit the measurement and transmission of vital health parameters and the exertion of externally controlled actions on the human body. Such systems provide evident benefits, but also pose significant new risks of misuse by totalitarian governments or criminals. In addition, "good" governments, in their effort to improve the lives of their citizens, may be tempted to rectify the conduct of those citizens, ignoring their wishes, and to enforce it with new means of total surveillance. This Global Brain, controlled by authorities advised by experts, and too complex to be overseen by the general public, may lead to a revival of the Rule of the Philosophers foreseen by Plato, a Brave New World where democracy is just an empty shell
\end{abstract}

Keywords: Pervasive Computing, Social Impact of Technology, Experts' Rule

Pervasive Computing is a new technological trend that will profoundly change our lives in the near future. This paper discusses the social consequences of this development. First, the technical possibilities of the sensor/actuator systems are presented. Then, a perspective on their beneficial uses is given, particularly in environmental studies, health service and medical research. The new applications are data intensive and collected data are often highly personal. Therefore the risks for privacy protection are discussed and some technical and organisational solutions are proposed. In this context the problem of data misuse for commercial and criminal purposes is usually considered. The focus of this paper is different - we will investigate the potential consequences of the use of the data by apparently benevolent public institutions.

\section{Sensors and Actuators Everywhere}

Gathering every available information about everything and everybody, supplemented by spatial and temporal data, allows society to build precise models of the physical world and of the human behaviour. With a little exaggeration the new systems can be said to form a sort of Global Brain, supporting decision centres with their data. This opportunity can be misused by a dictatorship, but also an overly caring government can interfere with the citizens' lives in an unpleasant way. The possible role of the experts in this Global Brain is discussed, their responsibilities and their temptations. Finally, some conclusions are presented - how the problems that arise could be solved by a community of noble researchers, honest leaders and educated citizens; and how to cope with them in a less ideal society.

The main idea of Pervasive Computing is to create an intelligent environment, enhanced by massively deployed computing and communications devices. Their task is to collect data about the real world and exchange those data between themselves or transmit them to a central computing system. Therefore pervasive computing is naturally associated with wireless sensor networks. It is however not limited to the sensor systems. Acting devices expand enormously its application range, enabling closure of the control loop and the exertion of action on the world.

We can divide the applications of pervasive computing into the following (neither exclusive nor exhaustive) groups:

- environmental

- medical

- vehicular

- surveillance

- smart grid

- building automation (and smart cities)

- mobile robots 
This trend can be exemplified by some scenarios. One variant of an environmental system may consist of a swarm of identical sensors covering a certain area with a grid of measurement points, delivering data for weather forecasting and for improving meteorological models. Another system may measure the pollution level and issue alarms in case of values which exceed the permitted limits. Still another can detect the seismic activity.

A personal medical system can measure health parameters and either deliver periodic reports to the user and to the hospital or send an alarm in case of an emergency. If an ambulance has to be dispatched, the geographical location of the patient is required. A medical system can also contain devices that act directly on the human body, such as to stimulate the heart action, inject insulin or administer medicine. Such a set of interacting wearable / implantable devices communicating over a short distance wireless network is called a Body Area Network. A local set can also communicate with remote systems, like hospital computing centres, with help of a smart phone or a similar device. Another example of a medical system is called Ambient Assisted Living. An example of this is a smart home that contains sensors detecting the patient's activities, and other devices providing him/her with various sorts of assistance in his/her daily life. Such a system is especially valuable for patients with non-acute chronic diseases, living alone. Health monitoring at home reduces the frequency and duration of hospital stays and delays the transfer to a nursing home.

The connection between sensors and actuators can be implemented in various ways. They can cooperate in a locally closed loop - for example in a vehicular network the sensors measure the distances between the vehicles and their speeds, and this information is directly transformed into the signals to the actuators that control the vehicles in order to prevent collisions. In a Body Area Network, the stroke detection software, fed by the sensors, recognises dangerous signal patterns and directly activates the pacemaker. The control loop can also be open and involve human operators. They analyse the information delivered from the sensor network using other information sources and their general knowledge, take decisions and act remotely via the deployed actuators or using other means. Sometimes the system's response requires a human action, as when a hospital is alarmed and an ambulance is dispatched.

This development has some basic enabling factors:

- miniaturisation of the electronic devices, which permits a massive deployment of them in an economically viable way;

- existence of a wireless telecommunications infrastructure with an almost comprehensive coverage: this makes possible a real-time, wide-area exchange of data;

- measuring the previously untreatable properties of chemicals, especially in interfacing with a fluid medium, which is important for medical applications;

- power management: extend the unattended lifetime of devices through new methods of efficient energy use, energy harvesting (or scavenging), and charging from an external source via wireless energy transfer.

This is not a future vision anymore - in December 2011, British prime minister David Cameron announced ${ }^{1}$ plans to install millions of health monitors ${ }^{2}$ in homes. This should vastly reduce the number of preventable death and emergency cases. The UK National Health Service intends to spend around $£ 750 \mathrm{~m}$ on such systems, but the expected savings are of $£ 1.2 \mathrm{bn}$ a year. The main goal is to improve the lives of individual patients, but of course there is a great potential for research if health data could be reused.

A broader, less technical view on the subject is given in two papers by Sliwa and Benoist: in one the ethical and social aspects (2011a) are discussed and in another the historical perspective (2011b) is presented.

\section{Researchers' Paradise}

This abundance of data is a paradise for researchers. Environmental sciences, climatology and biology are among its prime beneficiaries. Dense networks of sensors sending data allow researchers to study the dynamics of the weather system with a high precision. Individually marked animals disclose their migration routes and mating behaviour. Their eating habits and health condition can be observed.

Knowledge about the working of the human organism can be greatly enhanced. Accurate statistics of human behaviour are possible - such studies need not anymore to be based on unreliable

\footnotetext{
${ }^{1} \mathrm{http}: / /$ www.publicservice.co.uk/news story.asp?id=18224. Accessed 6 August 2012.

2 http://www.health-monitor.co.ukl. Accessed 6 August 2012.
} 
declarations. The connection between lifestyle, smoking, eating habits and health can be based on strong experimental evidence. The spread of contagious diseases can be tracked. Connecting sensor data with geographical data and further information about those involved such as their age, sex, marital status, education, job and income, can initiate truly experimental sociology and psychology. These sciences can also get a boost from the modern electrical power distribution system, the so-called smart grid. This may be surprising, as the main goal of the intelligent power meters is just to get data about energy consumption in order to optimize its production. However, the energy usage patterns reflect the daily rhythms of the users. As most of our actions are related to electrical devices, they tell when a water boiler or toaster was switched on and how many people took a shower.

In the European Union an important group of projects has been launched under the title Virtual Physiological Human ${ }^{3}$. Its goal is to model various aspects of human physiology, connecting basic research, clinical practice and computer models. As humans are not identical, not only a general model is to be determined - the aim of the project is also to find differences, for example, dependent on the genetic profile. This group involves many projects concentrating on measuring, modelling, simulating and predicting, with the general goal of obtaining a personalised medicine ${ }^{4}$.

In the US, the Mayo Clinic ${ }^{5}$ plans to sequence the full genetic code of thousands of people (Sample 2011) in a project to personalise their medical care, thus entering the era of "proactive genomics". One of the goals is to administer drugs corresponding to the genetic profile of the patient. In normal clinical practice a drug is prescribed, and it is only changed to another drug after no reaction or a side effect is detected. For example, a clot-busting drug Clopidogrel (or Plavix) helps most patients with heart problems, but for $20 \%$ has no effect. A single gene prevents its action, and this gene is known. With genetic analysis it is possible to select the adequate drug without wasting time and damaging the patient's health. As whole genome sequencing is now affordable, it is possible to analyse how multiple genes influence the progress of the disease and the reaction of the body to various drugs.

In order to use such methods, we first need to obtain knowledge in a number of areas. On one hand, the researchers try to understand the chemical basis of the process. On the other hand, these conjectures have to be verified by actual trials and statistics. If some cause-effect relationships are detected, it is necessary to know how certain they are and that they are not dependent on further factors. Therefore continuous monitoring is needed.

In the UK, the network of Public Health Observatories ${ }^{6}$ builds Health Profiles based on a set of indicators ${ }^{7}$, related to the health conditions, lifestyle, social situation etc. A map of dependencies is created (Jarman 2011) with two outcome measures, namely male and female life expectancy. The influences of individuals' factors are evaluated on the basis of comprehensive statistics, delivered by local authorities. The Health Profiles Indicator Guide ${ }^{8}$ contains detailed information (metadata) about the indicators and other data within the Health Profiles. It describes what, how and by whom is measured, what are the actual data sources, and what are the possible problems with collecting the data. This is important, because any bias or incompleteness of the data diminishes the value of the model. Tuning a network model is a complex mathematical problem. The dependencies are multidimensional; isolating causes and effects is not easy. Moreover, as the model is fed with data, the world is changing, so the parameters of the model vary with time. Life expectancy has subtle problems on its own - we can measure a lifespan only when someone already has died, and we are interested in the future lifespan of the persons living now. During a lifespan all external conditions change, so for example the correlation between smoking and life expectancy is influenced by many other factors. Even the cigarettes are not the same as before.

Therefore in this ocean of data, good navigation tools are necessary. Otherwise we risk the GIGO effect - garbage in, garbage out. Sheer data quantity may be misleading.

\section{Privacy Protectors' Nightmare}

Sensor networks produce immense amounts of data. Environmental data may be considered relatively harmless, but medical data are highly private. Even the information about power usage in the smart grid discloses details that should not be seen by strangers. In particular, the storage and processing of the health related data is strictly regulated. In Europe, the basic legislation is the

\footnotetext{
${ }^{3}$ http://www.vph-noe.eu/. Accessed 6 August 2012.

4 http://p-medicine.eu/. Accessed 6 August 2012

5 http://www.mayoclinic.org/development/individualized-medicine.html. Accessed 6 August 2012.

6 http://www.apho.org.uk/default.aspx. Accessed 6 August 2012.

7 https://indicators.ic.nhs.uk/webview/. Accessed 6 August 2012.

8 http://www.apho.org.uk/default.aspx?QN=HP USERGUIDE2011. Accessed 6 August 2012.
} 
Directive 95/46/EC on the protection of individuals with regard to the processing of personal data, and on the free movement of such data (Data Protection Directive ${ }^{9}$ ) with various amendments and national implementations; in the USA, it is the Health Insurance Portability and Accountability Act $\left(H I P A A^{10}\right)$. The main goal of these acts is the protection of personal data. Each collection of data should have a well-defined purpose, rules of access and retention. The rules are more flexible if data are implicitly necessary to provide a service to customers or if they help to maintain law and order. Secondary use is also permitted with the explicit consent of the concerned person. Health data may be used by health professionals when necessary for the provision of medical care to the concerned patient. An important aspect permits the processing of health data for the purposes of preventive medicine, medical diagnosis or management of health-care services, which opens the way for medical research.

It is evident that data for research are anonymised. Unfortunately, just removing names and addresses is not enough. If some facts about a person are known, it can be possible to find him/her using these. In case of medical research, if the device used by the patient is known, dates of medical interventions, behaviour patterns - all this can lead to a specific person. If the medical analysis includes age, gender, education, residence and social situation, finding the person is easy. In the aforementioned case of the Mayo Clinic, where genetic information is linked with the health records, even if all names are removed from data set, the genetic code can be used to pinpoint the patient. Of course, if a data thief wants to retrieve somebody's record on this basis there is a problem of getting a sample (e.g. saliva) and the cost of performing the genetic analysis.

There are methods to obfuscate the results delivered by the system, but important data cannot be removed if the research has to be of any value. Specifically, genetic research with distorted genetic data makes no sense at all.

The solution can be based on defining circles of users with different rights and responsibilities.

In the inner circle are the system operators. As insiders, they have access to the raw data. In their case, the solutions are psychological and social rather than technical, although separating data sets and allowing access only to partial data is a good approach. Also logging accesses and detecting doubtful operations is useful. If possible, data can be marked in order to trace the data leaks.

The researchers need all reasonable access to perform their studies. The problem is complex, because the legislators favour predefined data sets with clear usage, well isolated, with some data requiring special handling. The attitude of the researchers is the opposite of the legislators - connecting large, undistorted and complete data from many sources makes the analysis interesting. Similar problems are encountered in supporting medical registries. In the case of a medical followup study, if a patient does not respond to a call, this may be due to many reasons: he may be entirely cured and not interested in an inspection, he may be angry because of a wrong treatment and not wanting any contacts, he may have changed the address, he may even be dead because of a failed treatment or for another reason. If the researchers are not allowed to search for addresses and to access the death records, this crucial information is missing.

This does not mean that data access should be granted to everybody. The requirement of privacy protection has to be respected. Data sets have to be properly administered; the access to critical data has to be restricted to registered and verified users, bound by professional secrecy. This obviously contradicts the principle of free research, but human health records cannot be treated in the same way as data from a space telescope or a particle accelerator.

The general public has no need to see the detailed information. In any case, it is much better to deliver well presented and commented data. On the other hand, this raises a question of who should present these data and how they should do it, and the fear of manipulating data or hiding unpleasant facts is justified.

The important problem of commercial ownership of data is not discussed here, nor is the storage of data in an environment based on cloud computing.

\section{Big Brothers}

Ever since Pandora opened her box, curiosity has been a major driving force of the humanity. It is understandable that scientists and engineers want to work at the forefront or our technical capabilities. We need to consider the consequences of the new technologies. When helping to develop and deploy tools permitting a total knowledge and total surveillance we produce powerful, risky

\footnotetext{
${ }^{9}$ http://ec.europa.eu/justice/policies/privacy/law/indexl en.htm. Accessed 6 August 2012.

${ }_{10}$ http://www.legalarchiver.org/hipaa.htm. Accessed 6 August 2012.
} 
toys. We tacitly assume that we provide them for honest leaders, guiding a powerful group of democratic states. To what extent can we be sure of that?

We often think that with the fall of the Nazi regime and the decay of the Communism the case is closed, at least in Europe. This may be true in a relatively stable period of prosperity when there is enough to share. In harder times however, the hidden conflicts and the politically incorrect emotions, long swept under the carpet, may reappear. Any dreams of the end of history, of reaching an eternal paradise, are futile. Democracies do turn into dictatorships.

In a dictatorship, the roles change - the conspirators are the good guys. During World War II, in German-occupied Poland a decent clandestine laboratory could produce acceptable identity cards, it was possible to run a printing press in a cellar or to hide a Jewish neighbour - although at a high risk. Today's biometric passes are hard to forge and surveillance cameras together with smart power consumption sensors would detect the presence of undeclared machines or persons. Of course, if a large machine also made noise and increased the electricity bill, hidden persons could be seen by the neighbours and supplementary shopping in time of food rationing would in time become visible. The difference to our times is that in the 1940s a successful conspiracy relied on an active or passive cooperation of a relatively small group of people who would keep the secrets for themselves. Nowadays, the authorities using - or misusing, depending on your opinion - the detailed information coming from distributed sensors will be able to prevent any such actions.

What are the consequences? The new tools practically guarantee the superiority of the government over the citizens (although that may underestimate the citizens' ingenuity). If it acts in their interest, we are lucky. But what can we do with the dictators, as long as we are free to choose? We can decide not to deliver those tools to them, as we try to limit the access to nuclear weapons. But in many cases we will have to live with it.

\section{Caring Government}

Even a democratic government can care excessively for the well-being of the citizens, especially if it is guided by a grand idea of a radiant future, an ideal society or a New Human. The ideas of social engineering and eugenics were popular not only under Hitler and Stalin. Actually, the Nazi eugenics (racial hygiene, etc.) was inspired by the American program to create a master race (Black 2004). It was considered progressive and scientific. Shows were organised, e.g. Scientific Baby Contest at the Louisiana State Fair in Shreveport or Fitter Family competitions at the Kansas Free Fair. Even more or less active euthanasia on the feeble-minded has been practised. The movement - admitting its own political incorrectness - still lives on today ${ }^{11}$.

Social engineering has been also implemented in Switzerland. Some population groups were considered socially retrograded, among others the Yeniche. In the years 1926-1972, in the program called Kinder der Landstrasse ('children of the country road') (Galle and Meier 2009), some 600 Yeniche children were taken away from their parents and put into orphanages, mental institutions and even prisons.

As these bad experiences sink into oblivion, the idea of improving human beings reappears in other forms. For many, genetics opens the way for the Brave New World of designer children. Also the idea of correcting misbehaviours is back, especially if they are costly to the society. The case of smoking is clear, passive smoking is harmful to others. But obesity and unhealthy lifestyle are more and more considered not only as private problems. New tools can help to provide solutions.

As a possible scenario, let us imagine a research project that went out of control.

A group of researchers proposes a project having as a goal the study of human metabolic processes. New sensors that can be installed in the intestinal tract (implanted or swallowed) permit researchers to measure precisely the chemical composition of its contents. In this way the exact food intake is known, as well as the resulting products of the metabolism in various parts of the tract, pathogens and antibodies. Time-tagged measurements show the dynamics of the process. Body movement detectors complement the picture with physical activity patterns. No unreliable self-declarations are needed, all data are exact. Merging them with medical records shows consequences of lifestyle and nutrition for health.

After a certain time some conclusions may be drawn. As expected, some products lead to obesity, high blood pressure and heart diseases. Others cause cancer. This time however, the connection between causes and effects is not based on expectations; it is based on hard data, scientifically proven. As the results are promising, the scope of the project is expanded. The size of the sample is increased. As we know, the metabolism of various persons is not identical, it depends on genetics. Therefore genetic profiles are added, as well as geographic and social data, sex, age and

\footnotetext{
${ }^{11}$ http://www.eugenics.net/. Accessed 6 August 2012.
} 
education level. In this way it can be said not only that some people are susceptible to certain diseases, but which, when and how.

The results of the study spread to the general public. Not only alcohol and cigarettes are problematic, it becomes also evident that excessive consumption of chocolate is a major health threat. In addition, society is becoming conscious about the new measuring devices that can help to maintain good health. Metabolic analysers go into mass production. At first, only health conscious persons buy them on a voluntary basis; later, they are prescribed by doctors to obesity patients.

A debate begins, similar to that about tobacco - individual freedom vs. public good. Of course, passive chocolate eaters do not get any contagious disease, but they have to share the costs. Chocolate eaters are stigmatised as anti-social elements, they have to pay higher insurance rates. Every chocolate bar is adorned with a warning picture, finally chocolate consumption is banned.

As the now mass-produced metabolism testers get cheaper, their use becomes obligatory. No cheating is possible anymore. At first, there are protests against the secondary use of the information that originally served to enhance the medical research and serve the patients, but eventually the opinion prevails (with a little help), that public health and economy should be valued higher than protecting the bad habits of some individuals.

New devices perfectly suit individual drug control; this is their next application. They expand into other countries, where they are used to enforce the cultural or religious rules which forbid other substances, even some which are allowed in the parts of the world where they have been developed. Again, this new use generates protests, but the tools are readily available, it is not possible anymore to effectively control their use.

In the next step, the systems are complemented by acting devices. There are variants of them, depending on the severity of the case - some just preach into earphones, some give little shocks after a second chocolate bar per day, some act on the brain circuits, some call for human assistance.

If we look back, we see some analogies. We drive on predefined routes, we stop at the red light, we even fasten the seatbelts, although some still see it as violating personal freedoms. As we live in a society, in densely populated cities, we accept many rules. Pet animals have chip implants, why not us? A law abiding citizen has nothing to hide. Maybe we will accept total surveillance, even inside our bodies, with little helpers correcting the weakness of our will. We just have to be aware of the coming changes.

\section{Plato Redivivus - the Experts' Rule}

\subsection{No Democracy in the Airplane}

On a ship, the word of the captain is law. The same is true in an airplane. The passengers can choose their destination, select the menu and the movie, but control of the flight is in the hands of the pilot. They are not expected to discuss about thrust and the position of the ailerons. They accept that for their own good it is best to rely on the pilot's skills. Similarly at a lower level, the pilot relies on the software of the autopilot. The calculations are too complex, the necessary reaction times too short.

Are we in an airplane? Some would argue that in order to face the global challenges, we have to take coordinated actions orchestrated by people who know better. One of these challenges is global warming, known recently as climate change, which leaves the option of global cooling open. Currently (at the end of 2011) another such challenge is the financial crisis. It is suggested that we act fast and do not ask too many questions.

This reasoning recalls Plato's idea of the philosopher-king, the optimal ruler, presented in his "Republic". Having a bright mind and good memory as the basis, he (it never is she for Plato) searches for the very truth of each thing and he finds it. He loves the truth, and this noble attitude provides his soul with further virtues, like truthfulness, magnificence of mind and justice. He is absorbed in the pleasures of the soul, bodily pleasure do not distract him. The affairs of the State should be entrusted to men like him.

Today we know more. Popper (2002/1957) saw a corrupted version of the Plato's idea as the origin of modern totalitarianism and social engineering, the brave new world of Hitler and Stalin.

\subsection{Ivory Tower}

The fears of Popper may be exaggerated, but the problem of the interaction between science and power exists, in a similar way that previously the alliance of throne and altar corrupted the 
faith. Living in an ivory tower, in a feeling of intellectual superiority, connected with real power, may cause troubles.

One possible problem is fixing on the solutions and forgetting the goals. Goldstein (2011) presents the consequences of the advances in genetic screening of hereditary diseases. The genetic basis of more and more diseases is known, and a genetic test is also affordable to many parents. As most parents want to have happy, healthy children, this gives them more choice in what they pass on to their children. Technology does not permit the selection of a successful heir of the family business nor a man of honour, but allows parents to eliminate candidates with a known disease and to choose sex and eye colour, which would be feasible but is typically not permitted. Eventually, the main application is reducing the frequency of known diseases. As the ultimate goal is the quality of life of the children and their families and it is determined by many factors, this fixing on just one aspect of the problem is based on some doubtful assumptions.

The first assumption is that a disease is the worst thing that may happen to a person and to his/her relatives. It will make him/her unhappy to a degree that in his/her name we are allowed to choose the nonexistence. It seems that some personality traits, like egoism, narcissism, cheating or bullying may be worse. Unfortunately, they cannot be detected.

Then, every disease needs to be eradicated. In their zeal, the genetic screeners together with perfectionist parents want even to combat late onset diseases, like Huntington's and Alzheimer's Diseases. This means that 60 or 70 years of happy life are worth nothing if its end phase is marked by a predictable disease. But what is about the unpredicted diseases? Is the goal to win over all of them and to live eternally? It often can be read that one disease is eliminated, but unfortunately, people die of another one. It never was the other way round, and will never be.

Furthermore, there is no ethical dilemma. Abortion is hidden under euphemistic expression like disease screening and not transmitting mutations, which suggest that the whole problem is just a technical one and it is always a wise decision to protect society from un-aesthetic, needy and costly individuals, also for their own good. This perfect world would be free from van Gogh, Modigliani and other bipolar disorder patients. Stephen Hawking would not pass any screening.

And finally - we know what we are doing. Many diseases are not determined by a single gene. If many genes play a role, some of them may be active in other combinations, with other genes, causing other traits. Correcting a genetic problem may be similar to fixing a software bug without knowing the whole program - there is a good chance of introducing new bugs. It is important not to forget that we are mainly interested not in the genetic background but in effectively expressed traits, and here we can at best speak about probabilities. Moreover, some diseases may have positive effects in some cases: the gene for a blood disease called thalassaemia is widespread in the Mediterranean, e.g. in Sardinia, because the side effect of this disease is an increased protection against malaria. In this way, malaria provides a filter perpetuating the mutation. It may also happen that a pathogenic mutation is located next to another, positive one. If we concentrate on negative mutations, we can switch off the positive ones.

Another problem is seeing the numbers and forgetting the persons. Katsnelson (2011) describes the clinical trials for an experimental therapy of Parkinson's disease, called Spheramine. A crucial element is implanting some prepared cells into the brain. In order to test the therapy, and specifically to eliminate the placebo effect, double-blind test is necessary, where neither the doctor nor the patient knows who is receiving the real treatment. When a chemical is tested, it is enough to give some patients sugar pills; in case of a surgery, a fake (or sham) operation is performed - a real hole is drilled in the skull, but no substance is inserted. The studies have shown that the placebo effect is very strong - patients believing they have got the real treatment and hoping to improve, improve really, and not only in the short term. When they learn the truth, their condition rapidly deteriorates. What is then needed? A scientific attitude would suggest more tests in order to get better statistics. But is it the correct way? Of course, it makes no sense to perform expensive surgeries that have no effect - in this way the experiment is useful. But what is about the placebo effect? It is equally real, and explainable physiologically - positive feelings, hope induce the release of dopamine, the neurotransmitter that is lacking in the disease. For some, although the effect of placebo is higher than the effect of the therapy, real or fake, it is not the sort of answer that is expected, is not scientific enough. It has to be noted that fake surgeries are not everywhere considered harmless - they never have been used in the United Kingdom.

Generally, we have to remember what such terms like the mortality ratio in an experiment really mean. A mortality ratio of $10 \%$ in a sample of 300 patients means that 30 patients have died in course of the experiment. The experimenter should be sure that they have not been sacrificed in order to get better numbers. 
Still another problem of the experts' rule is their own Darwinist behaviour. If it is true that humans are optimized for survival, this applies also to the scientific community. University tenures and well-funded grants provide a pleasant ecosystem. Let us take climate research as an example. I do not intend to solve the scientific problem itself. The influence of the human activity on the climate seems to be proven. Is it however the dominant factor? We live in a climatic anomaly of a relatively stable climate over about twelve thousand years and we take this stability for granted. If we look at a longer history, for which oil companies take no responsibility, we see our present time as an interglacial period. As the periods are getting shorter - the previous interglacial lasted only for about 15,000 years, so maybe the next ice age is due. In the past, Earth was subject to many catastrophic climate changes, from a tropical jungle to an ice ball and back.

It may be possible to correct the minor climatic effects of the human activity, assuming that the system is otherwise fairly stable. Nevertheless, we should not underestimate its complexity. It is hardly imaginable that we can control all necessary parameters, even if we could agree on a worldwide coordinated action and weigh up the profits of some countries versus the losses of the others. Let the correction of the Australian ecosystem through the introduction of rabbits be a warning against simplistic manipulations of complex systems. Moreover, natural factors influencing our climate are orders of magnitude above our capabilities. To hope that we could ever stand up victoriously against earthquakes, volcanoes and variations of solar activity seems naïve. Facing such forces, we are in no better position than the ancient Egyptians. The project is however sold to the public as saving the climate. This can be understood that if we behave well, ride a bicycle and use low-power bulbs, the climate will be saved, which is not true.

Regarding human influence, how sure can we be of our models? Locally, the connection between the emission of $\mathrm{CO}_{2}$ and the greenhouse effect is understandable. But can we really predict the global effects? Only recently we have detected the influence of South-American El Niño on the weather worldwide. How many such effects are still undetected? Models are only models, they are similar to reality but not identical.

A real world problem should be solved by objective science. However, when we observe the discussion among the scientists, politicians and the general public, it is so highly emotional, that it seems that all are guided rather by their own interests. The scientific community has also lost some respect, when hacked e-mails have been disclosed (Revkin 2009). The case has been played down, but suggestions to correct unwanted data or discredit opponents have left a bad impression.

\section{Noble Researchers, Honest Leaders, Educated Citizens}

In the preceding sections, the new technologies have been described and the opportunities and dangers have been presented. We are now about to receive powerful tools to observe the world and to act upon it, a gift with a mixed blessing. In an ideal society, noble researchers gain objective knowledge about the world, including human beings, and use this knowledge for the benefit of society. Honest leaders manage the implementation of the reasoned solutions, they in their turn are controlled by educated citizens, who have a good understanding of the issues and trade-offs.

Our actual society is far from this ideal. The researchers, noble as they are, understandably follow more mundane goals, wanting to protect their common habitat and to promote their personal career. The Darwinian goal of the leaders in a democracy is to be (re)elected. Setting ambitious and difficult targets rarely serves this objective. The citizens follow rather their emotions than reason.

It has to be stressed that the very definition of the public benefit is far from being clear. Every decision has short term and long term effects. The effects of a decision are also different for various participants, so from another position a different solution will be seen as optimal.

In the context of a panoptic society, what we call here the Global Brain, the main line of conflict lies between personal freedoms and state power. Objectively, it can neither be said that the position of the state should always be strengthened nor that personal privacy should be valued higher. It depends on what kind of state it is and how the citizens behave, whereas in the case of a dictatorship considering moral principles to set the limits of the state's power does not have much sense, for obvious reasons. The optimal rules depend also on the actual situation - in case of an acute danger the state should be able to react quickly and efficiently.

But how to assess the danger? What is the value of preventing the risk of a terrorist plane hijacking? The consequences of such event are terrible, but how high is the probability? There were no major attacks after September $11^{\text {th }} 2001$, but were they prevented by stricter controls, were the possible attempts deterred by the controls or were there no attempts at all? Were the controls so efficient or useless? In some countries security has a top priority. In Switzerland, every house has 
a nuclear fallout shelter. There has however been no nuclear war so far. Was the effort lost? Not necessarily, as they can also be used in case of a natural or a technical disaster.

In a non-ideal society it is very difficult to discuss rationally about public issues. The issues are complex; even with the currently best science it is hard to solve them. Exact sciences were never easy. As early a writer as Proclus (1992/410-485) in his commentary on Euclid said: "There is no Royal Road to geometry"; and Barbie doll confirmed this with the memorable phrase "Math class is tough" ${ }^{12}$. Politicians are selected rather by their speaking abilities than by their understanding of physics. British scientist and novelist C. P. Snow (2001/1959) presented a growing chasm between the "two cultures" of human and natural sciences. He pointed that the question "Can you describe the Second Law of Thermodynamics?" is the scientific equivalent of "Have you read a work of Shakespeare's?", and "What do you mean by mass, or acceleration" is the equivalent of "Can you read?". Whereas illiteracy is shameful, many are even proud of their innumeracy. If there was any progress since the times of C. P. Snow, it was rather a progress of illiteracy - even among educated people a common saying like tempus fugit can rarely be used without explanation. The knowledge of a common heritage is eroding, partly because in a multicultural, liquid society (Bauman 2005) this heritage is less and less common. This does not mean that the society opposes science. On the contrary, science has many believers. Therefore it is useful to propagate an uncertain, biased message disguised as science. A nicely formatted infographic (McArdle 2011) is convincing, even if the data are manipulated and no credible sources are given.

In a democratic society an important factor is the public opinion. It is not a decisive one - with the exception of Switzerland, citizens only rarely have an opportunity to vote directly about specific issues. Nonetheless, their views have to be taken into account. How are those views formed, how is the consensus built? The citizens are not better informed than their leaders, but they are affected by the decisions, therefore they add the gut factor to the process. Couzin et al. (2011) argue that the uninformed individuals promote democratic consensus, because they follow the majority and isolate the extremists. Consensus has a value on its own, a hot conflict in a group is costly. A coordinated action is advantageous, but on the other hand, in the search for a solution, voicing openly different opinions is necessary. A common saying states, the truth lies in the middle, but in fact, the truth lies where it lies. A small minority may have a better understanding of the problem. It is a frequent plot of action movies - in Jaws ${ }^{13}$ only the main protagonists know the real danger, everybody else wants to pass a nice day on the beach. In real life a similar situation occurs, for example, when an influenza ${ }^{14}$ alarm is issued. A recent case was the 2009 pandemic. It was a popular subject in the media. But when it came to actions, opinions were divided. A preventive action consists of a quick development of a prevention drug, its mass production and a purchase by the authorities. The drug has been developed and produced; most developed countries bought a large supply, spending hundreds of millions of Euros. In the event, the virus was milder than predicted, and the stored drug was useless. In Poland however no drug was bought. At first, there was an outcry that the health minister was risking the people's lives, but eventually the high bet paid off. Who was right? Was the effort not necessary? After hearing experts cry wolf too often, with a panic magnified by the tabloids, many see the danger as overstated, rather like global warming/cooling/flood/earthquake as seen in the movies. But the danger of influenza is looming. The hard fact is that in 1918 the Spanish flu killed millions, and a mutated virus can return. It cannot be overturned by any voting.

Nowadays, we are facing technical developments that seem to overwhelm us. Specifically, we are becoming elements of a global system, network nodes, observable, controllable and configurable from outside. We do not get lost anymore, neither can we hide. The future vision need not be demonic - the new technology is of course beneficial to many of us, otherwise it would not make such progress. But do we want all of this?

We see that the decision process in a democratic society is not particularly efficient. The main players are not well informed and guided by emotions or personal, short term interests. The undecided follow the majority, real or apparent. Legislation is slow compared to technological development. In the global Internet environment, applicability and enforcement of law is problematic.

However, even this flawed process is better than anything else. The only solution is an open discussion, well informed and unbiased, in which the interests and fears of all parties are treated seriously.

\footnotetext{
${ }^{12}$ http://www.wired.com/geekdad/2011/12/math-class-is-tough/. Accessed 6 August 2012.

13 http://www.imdb.com/title/tt0073195/. Accessed 6 August 2012.

14 http://www.who.int/influenza/en/. Accessed 6 August 2012
} 


\section{References}

Bauman, Zygmunt. 2005. Liquid Life. Cambridge: Polity.

Black, Edwin. 2004. War Against The Weak: Eugenics and America's Campaign to Create a Master Race. New York: Thunder's Mouth Press.

Couzin, lain, Christos Ioannou, Güven Demirel, Thilo Gross, Colin Torney, Andrew Hartnett, Larissa Conradt, Simon A. Levin, and Naomi E. Leonard. 2011. Uninformed Individuals Promote Democratic Consensus in Animal Groups. Science 334: 1578-1580.

Galle, Sara, and Thomas Meier. 2009. Von Menschen und Akten: die Aktion "Kinder der Landstrasse" der Stiftung Pro Juventute. Zürich: Chronos.

Goldstein, David B. 2011. Growth of Genome Screening needs Debate. Nature 476: 27-28.

Katsnelson, Alla. 2011. Experimental Therapies for Parkinson's Disease: Why Fake it? Nature 476: 142-144.

Jarman, I.H., T.A. Etchells, C. Perkins, M.A. Bellis, and P.J.G. Lisboa. 2011. Scenario Analysis for Local Area Life Expectancy using Conditional Independence Maps. In Proceedings of the 4th International Conference on Developments in eSystems Engineering (DESE), Dubai, edited by Saad Ali Amin, Abir Hussain, Hissam Tawfik, Dhiya Al-Jumeily, Atulya K. Nagar, and Omar Abuelmaatti, 92-97. IEEE.

McArdle, Megan. 2011. Ending the Infographic Plague. The Atlantic, December 23. Accessed January 1, 2012. http://www.theatlantic.com/business/archive/2011/12/ending-the-infographic-plague/250474/

Popper, Karl. 2002/1957. The Poverty of Historicism. London: Routledge.

Proclus. 1992/410-485. A Commentary on the First Book of Euclid's “Elements", 57. Princeton University Press.

Revkin, Andrew C. 2009. Hacked E-Mail is New Fodder for Climate Dispute. New York Times, November 20. Accessed December 26, 2011. http://www.nytimes.com/2009/11/21/science/earth/21climate.html

Sample, Ian. 2011. Mayo Clinic Plans to Sequence Patients' Genomes to Personalise Care. The Guardian, December 28. Accessed December 30, 2011. http://www.guardian.co.uk/science/2011/dec/28/mayo-clinic-genomes-personalised-care

Sliwa, Jan, and Emmanuel Benoist. 2011a. Wireless Sensor and Actor Networks: e-Health, e-Science, e-Decisions. In Proceedings of the International Conference on Selected Topics in Mobile and Wireless Networking (iCOST), Shanghai, edited by Abderrahim Benslimane, 1-6. IEEE.

Sliwa, Jan, and Emmanuel Benoist. 2011b. Pervasive Computing - the Next Technical Revolution. In Proceedings of the 4th International Conference on Developments in eSystems Engineering (DESE), Dubai, edited by Saad Ali Amin, Abir Hussain, Hissam Tawfik, Dhiya Al-Jumeily, Atulya K. Nagar and Omar Abuelmaatti, 621-626. IEEE.

Snow, C.P. 2001/1959. The Two Cultures. London: Cambridge University Press.Acquisti,

\section{About the Author}

Jan Sliwa

received his MSc degree in Automation in 1977 from the Silesian Institute of Technology in Gliwice, Poland. After 4 years at an Institute of the Polish Academy of Sciences he moved to Switzerland where he developed software in languages ranging from Assembler to Java. He worked many years in the area of industrial control and then switched to Web-Database applications. In recent years his field of activity has been related to medicine (drug approval and medical registries). He is currently affiliated to the Department of Engineering and Information Technology of the University of Applied Sciences of Berne (BFH-TI) in Biel, Switzerland. His non-IT speciality is languages and cultures: Germanic, Romanic, Slavic and East Asian. 